
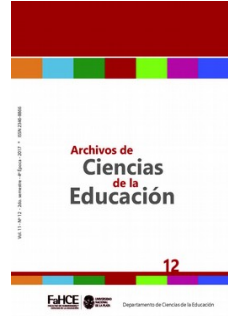

Archivos de Ciencias de la Educación, Vol. 11, n 12, diciembre 2017, e032. ISSN 2346-8866 Universidad Nacional de La Plata.

Facultad de Humanidades y Ciencias de la Educación.

Departamento de Ciencias de la Educación.

\title{
Políticas educativas para la educación rural en territorio bonaerense
}

\author{
Educational policies for rural education in the province of Buenos \\ Aires
}

\section{Mónica Escobar}

Universidad Nacional de La Plata, Argentina | moienero10@gmail.com

\section{PALABRAS CLAVE}

Políticas educativas

Políticas curriculares

Educación rural

Plurigrado

\section{KEYWORDS}

Educational policies

Curricular policies

Rural education

Multigrade

\section{RESUMEN}

En este artículo se presentan los hitos más destacados de las políticas educativas y curriculares que enmarcan a la educación rural de la provincia de Buenos Aires. Estas políticas son consistentes con los acuerdos suscriptos en la Conferencia Mundial (Jomtien, 1990), el Encuentro "Conocimiento y equidad” de la CEPAL (1992) y el Foro Mundial sobre "Educación para Todos” (Dakar, 2000). Como plantea Záttera (2015) estos encuentros internacionales se han constituido en antecedentes claves para el movimiento internacional de estudio y mejoramiento de la educación rural producido en la última década, exhortando a los países a proponer alternativas educativas para la atención específica de poblaciones vulnerables, entre las que se mencionan explícitamente las de zonas remotas y rurales.

\begin{abstract}
This article summarizes the most important milestones about educational and curricular policies that delimit the rural education in Buenos Aires Province. These policies are consistent with the subscripted agreements in the World Conference (Jomtien, 1990), the CEPAL (1992) "Knowledge and equity" meeting and the "Education for Everyone” World Forum (Dakar, 2000). According to Záttera (2015), these international meetings were key precedents in the last decade for the international movement for rural education study and improvement, exhorting the countries to propose educational alternatives for the specific assistance of vulnerable population, among which are explicitly mentioned those of remote and rural areas.
\end{abstract}




\section{Introducción}

Las escuelas primarias en Argentina se han conformado históricamente como instituciones organizadas en grados, en cada uno de los cuales se matricula a alumnos de la misma edad que reciben una instrucción simultánea a cargo de un maestro. Este formato original intentaba extender la presencia de escuelas primarias para posibilitar el acceso a la escolaridad obligatoria a gran escala. Ahora bien, por la misma razón, las escuelas también debían instalarse en aquellas zonas alejadas y de población dispersa en las que la baja matrícula impedía o dificultaba la organización graduada de sección única, dado que no era ni posible ni rentable sostener tantos cargos docentes para tan pocos alumnos. Las aulas plurigrado, en las que un maestro está al frente de un grupo de estudiantes que cursan diferentes años de la escolaridad, ha sido una de las respuestas a esta problemática.

En la actualidad, la provincia de Buenos Aires cuenta con un total de 4.249 escuelas primarias, de las cuales 2.649 son urbanas, 1.600 rurales (1.572 continentales y 28 de islas). ${ }^{1}$ Dentro de las escuelas rurales y de islas hay 40 en las que la matrícula es de 1 alumno y 865 a la que asisten entre 2 y 10 alumnos. $\stackrel{2}{\text { Pese a esta gran }}$ diversidad de realidades, tanto la formación docente inicial y continua como las propuestas curriculares y editoriales, han dado escasas respuestas específicas para las diversas modalidades de organización entre las que se encuentran las aulas plurigrado - tan frecuentes en las escuelas rurales y de islas-. Sin embargo, es justo señalar que en los últimos años se han producido algunos cambios.

La Conferencia Mundial (Jomtien, 1990), el Encuentro “Conocimiento y equidad” de la CEPAL (1992) y el Foro Mundial sobre "Educación para Todos” (Dakar, 2000) resultan antecedentes claves para el movimiento internacional de estudio y mejoramiento de la educación rural producido en la última década. Los acuerdos suscriptos en estos encuentros internacionales exhortaron a los países a proponer alternativas educativas para la atención específica de poblaciones vulnerables, entre las que se mencionan explícitamente las de zonas remotas y rurales (Záttera, 2015).

En este artículo presentaremos los hitos más destacados de las políticas educativas y curriculares que han enmarcado a la educación rural de la provincia de Buenos Aires. Para ello, retomamos el relevamiento realizado en el marco de un estudio reciente sobre la enseñanza de la matemática en aulas plurigrado. ${ }^{3}$ Sin pretensión de exhaustividad, trazaremos un recorrido histórico de la escuela primaria en la Argentina y en la provincia de Buenos Aires hasta nuestros días haciendo especial referencia al origen del aula plurigrado como modalidad de organización característica de las escuelas rurales, a sus continuidades y transformaciones a lo largo de estos más de cien años de historia. Comenzaremos haciendo una breve referencia a la Ley 1420 y a las políticas educativas destinadas a la educación rural iniciadas en los años 90, haciendo foco posteriormente en las reformas normativas y curriculares que se sucedieron entre 2006 -año en que se promulga la Ley Nacional de Educación- y 2015. A su vez, haremos referencia a la producción curricular, la formación profesional y las condiciones de trabajo docente en el ámbito rural.

\section{La educación rural a través de los marcos normativos}

En los inicios del sistema educativo argentino la Ley de Educación $N^{\circ} 1.420$, sancionada en 1884, declara que la escuela primaria debe ser obligatoria, gratuita y graduada (art. 2). Si bien esta ley es la primera de alcance nacional en materia educativa, "una gran expansión de la cobertura (de escuelas primarias) tuvo lugar en las décadas de 1850 y 1860” (Záttera, 2015, p. 30). La Constitución Nacional de 1853 delegaba en las provincias la responsabilidad de asegurar la educación primaria (art. 5). Olga Záttera advierte que si bien el texto de la Constitución no se refería explícitamente a las escuelas rurales, como la población rural superaba el 60\% del total del país, es posible afirmar que formaban parte del universo de escuelas de la época. La autora expresa: “Crear escuelas significaba instalar locales donde se pudiera enseñar y un maestro 
a cargo podía resultar suficiente. En los orígenes de los sistemas educativos un maestro a cargo de todos los alumnos era 'el' modelo” (p. 31).

Por su parte, Inés Dussel (2006) señala que antes de la organización del sistema educativo común establecida por la Ley 1.420 había distintas escuelas “elementales” que no seguían un criterio único ni un mismo programa, entre las que menciona escuelas particulares, mixtas, para huérfanos, de ciudad y de campaña. Esta ley recupera aquellos primeros años de experiencia e instala el mandato de obligatoriedad, gratuidad y gradualidad que se ha extendido hasta nuestros días.

Junto con la prescripción de obligatoriedad, la ley establecía para la enseñanza primaria la división en seis o más agrupaciones graduales sin alteración de grados (art. 9). En aquellos casos en que la concentración de población lo hiciera posible, cada uno de los grados estaría a cargo de un maestro. En territorios de población dispersa, un maestro a cargo de todos los grados permitiría al mismo tiempo dar cumplimiento a la obligatoriedad y racionalizar la asignación de cargos docentes. ${ }^{4}$ Incluso el texto de la ley prevé para las zonas de población muy diseminada, la creación de "escuelas ambulantes, en las campañas, donde no fuese posible establecer con ventaja escuelas fijas” (art. 11).

Záttera (2015) resalta que desde los orígenes del sistema educativo hubo una preocupación manifiesta por pensar tanto en la escuela rural como en la escuela urbana, no solo al buscar aquellos formatos escolares que hicieran posible la instalación de escuelas a gran escala en todo el territorio, sino también al distinguir los contenidos de enseñanza que se abordarían en los diversos contextos. Entre los contenidos mínimos establecidos para la instrucción obligatoria de la escuela primaria se destacaban lectura, escritura y aritmética, e incorporaban específicamente para la campaña nociones de agricultura y ganadería (art. 6). $\underline{5}$

En relación con la Ley Federal de Educación N 24.195 sancionada en 1993ㅜㅡ, el Consejo Federal de Educación (CFE) -conformado por los Ministros de Educación de todas las provincias- aprobó en 1994 los Contenidos Básicos Comunes (CBC) para el Nivel Inicial y la Educación General Básica (EGB). Los CBC definían los saberes relevantes a partir de los cuales cada jurisdicción debía elaborar sus diseños o lineamientos curriculares considerando cada realidad regional. A su vez, a partir de un nuevo nivel de contextualización, cada escuela debía formular su Proyecto Curricular Institucional. En relación con los CBC y los Diseños Curriculares Provinciales, el Ministerio de Educación, Ciencia y Tecnología (MECyT) declara en un informe presentado en Santiago de Chile (2004) que "no se han definido para la EGB contenidos diferentes para zonas urbanas y rurales sino que se promueve que en la definición de los proyectos curriculares institucionales se tenga en cuenta el contexto local” (p. 4). A continuación, se retomarán algunos de los aspectos de este informe. Es posible reconocer en ellos ciertos planteos que pueden interpretarse como antecedentes de la Ley 26.206 de Educación Nacional sancionada en 2006.

El informe parte de una caracterización de las escuelas instaladas en zonas rurales con la intención de describir la problemática identificada. Luego, detalla las acciones implementadas por el MECyT en respuesta a las necesidades relevadas y delinea los desafíos pendientes.

La descripción de las escuelas rurales resalta su carácter de lugar de referencia para la comunidad, la particularidad del modelo organizacional del plurigrado (mencionando tanto su potencialidad pedagógica como su complejidad), la soledad y aislamiento del trabajo del docente, la escasez de propuestas que consideren la especificidad de la ruralidad en la formación docente inicial y continua o en propuestas de desarrollo curricular, el ingreso tardío de los alumnos y la discontinuidad de la asistencia. En relación con este último aspecto, se expresa:

Es frecuente que los niños ingresen tardíamente a la escuela, entre otros aspectos, por la escasa oferta educativa para el Nivel Inicial, por las condiciones de vida particulares de las familias y por las grandes distancias a recorrer para llegar a la escuela. En diferentes momentos del año un 
importante número de niños y jóvenes se ausenta de la escuela por períodos prolongados, por razones climáticas, por incorporación a trabajos temporarios junto a sus familias o por tener que cuidar a sus hermanos menores en ausencia de sus padres (MECyT, 2004, p. 9).

El MECyT, dando continuidad a acciones previas y en respuesta a las necesidades relevadas, crea en 2004 el Área de Educación Rural, $\underline{7}$ con el propósito de brindar una atención específica a la educación de la población rural. Este organismo plantea la necesidad de considerar al universo de escuelas rurales en forma conjunta sin perder de vista las particularidades.

Dar respuesta a las necesidades educativas de zonas rurales requiere tomar en consideración estas regularidades, de modo de identificar los aspectos comunes para la definición de políticas educativas orientadas al conjunto de la población rural. Pero también es necesario reconocer los aspectos diferentes, la diversidad de situaciones que suelen identificarse bajo la generalizada denominación de ruralidad, para encontrar respuestas situadas, adecuadas a distintos contextos (MECyT, 2004, p. 9).

El desafío de la consideración simultánea de los rasgos particulares y universales no se circunscribe al colectivo de escuelas rurales sino que atañe al diseño e implementación de políticas educativas dirigidas al conjunto del sistema educativo.

Por otra parte se considera que atender la ruralidad desde una perspectiva transversal no implica constituir un subsistema rural en cada jurisdicción, sino considerar las particularidades de la educación rural cuando se determinan líneas de política educativa nacional y provincial, para el conjunto del Sistema (MECyT, 2004, p. 11).

Frente a la identificación de la escasez de propuestas específicas vinculadas a la ruralidad, el MECyT coloca a la formación docente inicial y continua dentro de las líneas prioritarias e impulsa acciones articuladas con los ministerios provinciales destinadas al mejoramiento de las prácticas docentes en estos contextos.

La incorporación de la perspectiva de la ruralidad en la formación de base desde cada una de las asignaturas en las que se hace necesario considerar las particularidades del contexto en el que se desarrolle la actividad docente y desde las instancias de práctica y residencia, aportando experiencias de docentes rurales. (...) Propuestas de capacitación relacionadas con la enseñanza en plurigrado, que posibiliten la tarea conjunta de los docentes de escuelas próximas a partir de temáticas comunes y que remitan a realizar experiencias en las aulas de grados múltiples promoviendo la conceptualización a partir de ellas. Por otra parte se está revisando la necesariedad y características de post-títulos de enseñanza en la ruralidad (MECyT, 2004, p. 15).

Más adelante, especifica que atender las particularidades del contexto involucra reflexionar sobre la particular modalidad de organización del plurigrado.

Implica considerar al plurigrado como eje del trabajo en las propuestas de formación docente inicial y continua. Contemplar el plurigrado requiere abordar cuestiones institucionales, organizacionales, curriculares y didácticas. Es frecuente en los plurigrados que las propuestas de enseñanza se planteen en simultáneo y de manera independiente para cada año de escolaridad. Se trata de capitalizar la riqueza que ofrece el trabajo conjunto entre niños y/o jóvenes de diferentes edades, atender a la diversidad curricular que es necesario considerar simultáneamente y reconocer cierta unidad en el trabajo a partir de identificar los aspectos comunes. Se contempla en estas propuestas lo propio de la gestión institucional en escuelas con grados agrupados, junto a alternativas de situaciones de enseñanza en las que se dote de sentido al trabajo en grupos diferenciados conformados a partir de criterios que excedan el año de 
escolaridad, por ejemplo, dando lugar al trabajo compartido en función de las necesidades de aprendizaje, o por intereses afines, edades próximas independientemente del año de matriculación, contenidos similares desarrollados con diferentes grados de complejidad, recursos comunes para avanzar en el tratamiento de contenidos diferentes, etc. (MECyT, 2004, p. 17).

Al avanzar en la descripción de las líneas estratégicas propuestas por el MECyT para la gestión 2004-2007 en vistas a mejorar la educación rural, el mismo informe manifiesta la necesidad de superar el aislamiento de maestros y alumnos a partir de establecer vínculos entre escuelas y entre la escuela y la comunidad. Se plantea la "organización de agrupamientos de escuelas cercanas como una forma de posibilitar el diseño de propuestas comunes para mejorar las condiciones de enseñanza y aprendizaje en todos los ciclos y niveles” (p. 16), iniciativa sostenida hasta la actualidad. Con la intención de optimizar los materiales disponibles, en cada agrupamiento se promueve la conformación de centros de recursos posibles de ser compartidos por el conjunto de escuelas de una misma zona.

Por último, interesa retomar de este informe la cuestión de la flexibilidad etaria que resulta un antecedente relevante tanto para la Ley 26.206 (2006) como para el Diseño Curricular para la Educación Primaria (en adelante DCEP) (2008) y el Régimen Académico del Nivel Primario (2014) de la Provincia de Buenos Aires.

Es imprescindible considerar que a partir de los trece años los niños suelen dejar la escuela independientemente del año $\underline{\underline{8}}$ al que asistan; los altos índices de sobreedad motivan que un número importante de alumnos no complete la EGB2. Para los alumnos con sobreedad se promoverán alternativas que, en el marco de plurigrados, permitan a los jóvenes completar la EGB2 en menos de tres años, desde propuestas de enseñanza acordes a sus necesidades. Fortalecer los procesos de aprendizaje en la EGB2 seguramente permitirá mejores condiciones de acceso al Tercer Ciclo como un camino para garantizar la continuidad de la trayectoria escolar de los alumnos de escuelas rurales (MECyT, 2004, p. 18).

Es importante destacar la intención de habilitar a los alumnos con sobreedad a cumplimentar el segundo ciclo de la EGB en menos de tres años. $\underline{9}$ Esta habilitación es coherente con las propuestas de flexibilización de los formatos escolares que se plasmaron progresivamente en la normativa educativa y en diversos programas implementados en nuestro país. Un ejemplo es la Resol.174/CFE/12 en la que se identifican el ingreso tardío, los abandonos, las repitencias (art. 2) y las propias lógicas de escolarización (art. 3) entre los principales factores que obstaculizan la continuidad de las trayectorias escolares de los estudiantes de los niveles inicial y primario. En consecuencia, se señala el carácter prioritario de la búsqueda de diferentes...

...formas de escolarización que permitan superar prácticas y saberes que esperan lo mismo, de la misma manera y al mismo tiempo, de todas y todos los estudiantes. Parte de las dificultades en la trayectoria escolar se vinculan con la existencia de formas rígidas de organización que responden a un modelo escolar homogeneizador. Este modelo dificulta el trabajo con la heterogeneidad, excluye educativamente a muchos estudiantes y devalúa la propuesta escolar para el conjunto (art. 4).

Por último, esta resolución refiere diversas acciones implementadas entre las que se destacan los proyectos de reingreso y los programas que atienden a la sobreedad $\underline{10}$ basados en la reorganización del agrupamiento escolar (art. 6).

A continuación, se hará referencia a la Ley 26.206 que consagró la obligatoriedad escolar desde el nivel inicial hasta el nivel secundario completo y reestructuró los niveles educativos del sistema. $\frac{11}{1}$ Se hará hincapié en aquellas cuestiones que impactan especialmente sobre las escuelas primarias rurales. La creación 
de la Modalidad Educación Rural permite encuadrar el desarrollo de políticas universales destinadas a la totalidad (y diversidad) de escuelas rurales. El Capítulo X de la Ley está destinado a esta Modalidad y a través de los artículos que lo componen el Estado Nacional se compromete a garantizar el cumplimiento de la escolaridad obligatoria a través de formas adecuadas a las particularidades de la población rural (art. 49) y a arbitrar los medios necesarios para dar cumplimiento a los objetivos puntualizados para la educación en estos contextos (art. 50). Transcribimos a continuación dos de estos objetivos:

Garantizar el acceso a los saberes postulados para el conjunto del sistema a través de propuestas pedagógicas flexibles que fortalezcan el vínculo con las identidades culturales y las actividades productivas locales $\underline{12}$. (...)

Permitir modelos de organización escolar adecuados a cada contexto, tales como agrupamientos de instituciones, salas plurigrados y grupos multiedad, instituciones que abarquen varios niveles en una misma unidad educativa, escuelas de alternancia, escuelas itinerantes u otras, que garanticen el cumplimiento de la obligatoriedad escolar y la continuidad de los estudios en los diferentes ciclos, niveles y modalidades del sistema educativo, atendiendo asimismo las necesidades educativas de la población rural migrante.

Luego de la sanción de la Ley 26.206, en el año 2007 se sanciona la Ley de Educación de la Provincia de Buenos Aires $N^{\circ}$ 13.688. Dicha Ley establece una escuela primaria obligatoria de seis años de duración a partir de los 6 años de edad (art. 27). Estos seis años se incluyen dentro de un total de catorce años de obligatoriedad que abarcan desde la sala de 4 años de Nivel Inicial hasta $6^{\circ}$ año de Nivel Secundario. El contexto rural se menciona como uno de los ámbitos de desarrollo de la educación (art. 46). $\underline{13}$ Más adelante plantea la necesidad de definir modelos de organización escolar adecuados a la diversidad de los ámbitos rurales continentales y de islas a través de propuestas pedagógicas flexibles que fortalezcan el vínculo con las identidades culturales y las actividades productivas promoviendo el desarrollo de la comunidad (arts. 48 y 49).

El documento "Educación Rural en el Sistema Educativo Nacional” (2010) presentado por el CFE para ser debatido antes de su aprobación $\underline{14}$ es otro hito significativo en el sostén de la atención a esta problemática. El CFE retoma y avanza en el tratamiento de cuestiones planteadas por el MECyT y referidas en páginas anteriores: la particular organización del plurigrado como respuesta a la matrícula reducida típica de las escuelas rurales, la escasa conceptualización pedagógica de los saberes construidos por los docentes y la insuficiente presencia de contenidos vinculados con los contextos rurales en la formación inicial y continua. Asimismo, reconoce la necesidad de poner en tensión la naturalización de la escuela graduada urbana como modelo homogéneo y de promover estudios acerca de la escuela rural. El plurigrado y la conformación de agrupamientos son propuestos como ejes que articulan la definición de políticas para la ruralidad.

Finalmente, cabe hacer mención al Régimen Académico del Nivel Primario vigente en la provincia de Buenos Aires desde 2014, $\underline{15}$ destinado a regular las trayectorias educativas en la Educación Primaria en lo atinente a: inscripción, asistencia, evaluación, acreditación, calificación, promoción, movilidad y egreso, así como a la organización de las instituciones, su convivencia y comunicación. Desde el punto de vista conceptual esta norma se asienta en los principios de obligatoriedad escolar; inclusión con aprendizaje; promoción y protección de los derechos de niños, niñas y adolescentes establecidos tanto por las Leyes 26.206 y 13.688 como por los tratados internacionales de protección y respeto por los derechos humanos. En relación con las pautas de matriculación, este marco normativo reconoce y habilita la inscripción fuera de término -tan frecuente en las escuelas rurales- con la intención de garantizar la inclusión y el inicio o continuidad de las trayectorias educativas de los alumnos. A su vez, establece que en caso de ingreso tardío o reingreso se evaluará la posibilidad de matricular al alumno en el grado/año correspondiente a su edad. En 
estas circunstancias, "la escuela se comprometerá a generar los dispositivos pedagógicos para que el alumno logre, en el menor tiempo posible, alcanzar los contenidos del año que correspondan a su edad” (Capítulo II, Pauta de matriculación $N^{0}$ 12). Por otro lado, el Régimen Académico señala que la escuela y la familia deberán organizarse de manera conjunta en pos de sostener la regularidad de la asistencia para evitar interrupciones en las trayectorias escolares de los alumnos.

Los diversos marcos legales y normativos repasados hasta el momento han posibilitado la configuración de la educación primaria rural en la provincia de Buenos Aires. A continuación, se avanzará en la descripción de los contextos curricular, formativo y laboral.

\section{La educación rural y la enseñanza en aulas plurigrado desde el marco curricular}

A partir de las leyes 26.206 y 13.688 la Provincia de Buenos Aires aprobó en 2008 el Diseño Curricular para la Educación Primaria, vigente hasta la actualidad. Se trata de un diseño curricular único que regula la propuesta educativa del nivel primario incluyendo los diferentes ámbitos y modalidades del sistema educativo provincial.

Este diseño se dirige, particularmente, a todas las escuelas de educación primaria de la Provincia de Buenos Aires, en tanto es responsabilidad de las definiciones curriculares ofrecer el marco de educación común que garantiza el derecho de los alumnos/as a acceder a los saberes socialmente reconocidos como relevantes (DCEP, 2008, p. 28).

En el Marco General del DCEP se explicita la toma de posición respecto de la concepción pedagógica y didáctica asumida desde la política educativa provincial. Si bien no se desarrollará aquí la totalidad de su contenido, se tendrán en cuenta ciertos tópicos como marcos referenciales para la educación rural.

En el DCEP se valoran la continuidad de la asistencia a clase y de la propuesta de enseñanza, procurando presentar a los alumnos situaciones que desafíen sus conocimientos y los hagan progresar. En el contexto rural, la continuidad de la asistencia suele estar interferida tanto para alumnos como para docentes por factores climáticos y de accesibilidad, y en el caso de los alumnos por la organización familiar del trabajo y del cuidado de los hermanos. Esta situación particular suele afectar la continuidad de la enseñanza y de los aprendizajes.

En consonancia con los marcos normativos desarrollados en el apartado anterior, el DCEP se pronuncia a favor de la diversidad a partir de discutir posturas homogeneizantes de la enseñanza que suponen que todos los niños deben aprender idénticos contenidos al mismo tiempo. $\frac{16}{}$ A su vez, avanza en asumir la heterogeneidad (de conocimientos, de modos de organizar las clases, de culturas y de significados) no solo como realidad constitutiva de cualquier aula sino también como ventaja pedagógica. Si bien estas ideas están dirigidas a todas las escuelas, representan un desafío particular para los docentes que trabajan en aquellas organizadas en plurigrado. En estas aulas la diversidad de edades, conocimientos, contenidos y ritmos de aprendizaje se hace tan visible como inevitable (Broitman et al., 2016). En relación con este tópico del diseño curricular, transcribimos el siguiente párrafo del Marco General:

La escuela tal como la conocemos tiene características que subrayan la homogeneidad como rasgo relevante: un grupo de alumnos/as de una edad similar que cursan un mismo año, un mismo espacio, un mismo tiempo, un/a docente que desarrolla una propuesta de enseñanza para todos en forma simultánea, un ciclo lectivo completo para enseñar determinados contenidos cuyo dominio permitirá a ese grupo aprobar ese año y pasar al siguiente. Se exceptúan de esta descripción las escuelas rurales o de islas que, instaladas en comunidades pequeñas generalmente en situación de aislamiento relativo, atienden a matrículas reducidas que exigen una organización peculiar: alumnos/as matriculados en varios años diferentes comparten el tiempo y el espacio de aprendizaje conformándose plurigrados a cargo de uno, dos o tres 
docentes (DCEP, 2008, p. 21).

El fragmento citado reviste un interés particular por ser el único pasaje en el que se menciona al aula plurigrado dentro del DCEP. La conformación de la matrícula de estas aulas (integrada por alumnos que cursan diferentes grados de la escolaridad) torna evidente la diversidad de los contenidos de enseñanza y de los conocimientos infantiles que circulan en ellas. Esta realidad particular, según se expresa, las alejaría de la caracterización de las aulas estándar atravesadas por la homogeneidad.

Este currículo se presenta con idéntico formato y contenido ante docentes que se desempeñan en diferentes realidades. Los docentes de escuelas graduadas de sección única afrontan la tarea de planificar y conducir un proyecto de enseñanza pensado para niños de la misma edad -aunque sus conocimientos puedan ser diversos-. Sus propuestas didácticas se organizan, generalmente, en torno a la enseñanza de un mismo contenido para el conjunto de la clase. Los maestros de aulas plurigrado, en cambio, se enfrentan frecuentemente en soledad- a la lectura completa del diseño curricular en busca de aquellos contenidos pautados para los diversos grados de la escolaridad que cursan sus alumnos. Esta tarea requiere considerar diferentes edades, conocimientos y niveles de autonomía y buscar alternativas organizacionales que permitan gestionar la enseñanza en forma simultánea.

El DCEP presenta una organización por áreas (Prácticas del Lenguaje, Matemática, Ciencias Naturales, Ciencias Sociales, Educación Artística y Educación Física, incorporándose Inglés en el $2^{\circ}$ ciclo). La propuesta de cada área curricular incluye una selección y secuenciación de contenidos por ciclo $\left(1^{\circ}\right.$ y $2^{\circ}$ ciclos) ${ }^{\frac{17}{17}}$ o por año (de $1^{\circ}$ a $6^{\circ}$ ). Algunas áreas, como por ejemplo Prácticas del Lenguaje, presentan la selección de contenidos pautados para cada ciclo sin definir su distribución por año/grado. En cambio, otras, como por ejemplo Matemática, incluyen una selección y secuenciación de contenidos por año (de $1^{\circ}$ a $6^{\circ}$ ). Como ya se documentó ampliamente en la primera parte de este artículo, la producción pedagógicodidáctica, las propuestas curriculares y la formación docente presentan escasas referencias y aportes específicos para las escuelas rurales organizadas en plurigrados. Las orientaciones didácticas que acompañan a los contenidos propuestos por el DCEP no escapan a esa realidad y parecen no contemplar la singularidad de estas aulas.

\section{La educación rural y la enseñanza en plurigrado en la formación docente}

El estudio de la enseñanza en aulas plurigrado involucra necesariamente profundizar la mirada sobre la realidad histórica y presente de la formación docente inicial y continua. La indagación realizada sobre este aspecto permite identificar tanto las áreas de vacancia en relación con la especificidad del aula plurigrado en las propuestas formativas como los avances producidos a nivel provincial y nacional. Este apartado se organiza en dos partes: la primera referida a la formación inicial y la segunda, a diversas instancias de formación continua.

\section{Formación docente inicial}

La formación docente inicial en Argentina tiene su origen a principios del siglo XX. Era necesario formar maestros para cubrir la gran escala que requería el cumplimiento de la reciente Ley 1.420 de Educación y para ello se abrieron escuelas normales rurales mixtas en distintas regiones del país. Con el progresivo avance de la urbanización, estas escuelas -y más adelante los ISFD- quedaron incluidas en los nuevos espacios urbanos; esto trajo aparejado un progresivo cambio en los planes de estudio. Los contenidos de la formación docente ligados a lo rural comenzaron a ofrecerse en forma de seminarios optativos.

Los diversos estudios, informes y normativas reseñados en páginas anteriores, coinciden en señalar que a pesar del alto porcentaje de escuelas rurales plurigrado de nuestro país, $\underline{18}$ la formación docente inicial ha 
dado escasas respuestas específicas para esta modalidad de organización. Los maestros que se desempeñan en estas aulas han sido formados mayormente en ISFD cuyos planes de estudio se centran en la escuela urbana de sección única.

Tal como se ha referido, la Ley 26.206 define a la Educación Rural como una de las ocho modalidades del sistema educativo (art. 17) y explicita la necesidad de "desarrollar las capacidades y los conocimientos necesarios para el trabajo docente en los diferentes niveles y modalidades de sistema educativo" (art. 73). A partir de esta ley, se aprueban los "Lineamientos curriculares nacionales para la formación docente inicial" (Resolución 24/CFE/07), la resolución sobre "Titulaciones para las carreras de Formación Docente" (Resolución 74/CFE/08) y las "Recomendaciones para la elaboración de diseños curriculares. Educación Rural” (MECyT, 2009). $\underline{19}$

A su vez, la Resolución 74/CFE/08 define la nominación del título habilitante como "Profesor de Educación Primaria” para una carrera no menor a los 4 años de duración. Esta resolución retoma lo pautado por el art. 73 de la Ley 26.206, en el que se expresa la voluntad de "otorgar validez nacional a los títulos y las certificaciones para el ejercicio de la docencia en los diferentes niveles y modalidades del sistema”. La misma resolución aprueba para el Nivel Primario las siguientes orientaciones: Orientación en Educación Rural, Orientación en Educación Permanente de Jóvenes y Adultos, Orientación en Educación Hospitalaria y Domiciliaria, y Orientación en Educación Intercultural Bilingüe. Sin embargo, como lo explicita el artículo 129 de la resolución, la postitulación específica no resulta un requisito excluyente para la ocupación de los puestos de trabajo.

Los lineamientos y las recomendaciones curriculares para la formación docente inicial producidos por el MECyT apuntan a profundizar y mejorar las definiciones curriculares y fortalecer la integración nacional del currículo de formación docente (MECyT, 2007, art.6).

En la mayoría de las jurisdicciones de nuestro país, la formación de docentes para el nivel primario implica abordar las necesidades y particularidades del desempeño en escuelas de contextos rurales. Según lo explicita la ley de Educación Nacional (LEN) No 26.206 en su Artículo 11, su necesaria focalización no debe desconocer las indispensables regularidades que garanticen una educación común para todos los niños (MECyT, 2009, p. 9-10).

A partir de la Ley 26.206 y de la Ley 13.688, la provincia de Buenos Aires aprobó en 2008 el nuevo Diseño Curricular para la Formación Docente (en adelante DCFD) producido durante el año 2007. El detalle del orden temporal es relevante para señalar que su elaboración fue anterior a la aprobación de la Resolución 74/CFE/08, en la que se proponen las orientaciones específicas mencionadas anteriormente, incluida la de Educación Rural.

El DCFD está organizado alrededor de cinco Campos y Trayectos opcionales (DCFD, 2008, p. 29). El Campo de la Práctica Docente se considera el eje vertebrador y articulador del resto de los campos formativos.

En este Campo (de la Práctica) se propone que los 'maestro/as orientadores'르 de las Escuelas donde los maestro/as en formación realizan sus prácticas, participen en el Taller Integrador Interdisciplinario o en otras instancias del Campo de la Práctica Docente. Esta estrategia permitiría propiciar espacios de reflexión-acción y de construcción pedagógica en la zona de diálogo entre la cultura de la formación docente y la cultura escolar. Por otra parte, apunta a generar redes con las escuelas de la localidad y de las regiones de la jurisdicción, promovidas y articuladas con los Institutos Formadores (DCFD, 2008, p. 33). 
En relación con las escuelas destino, el DCFD establece que deben abarcar diferentes ámbitos y modalidades del sistema educativo.

Además, las escuelas donde se realicen las prácticas deben responder a diferentes características, no solo geoambientales, sino también socioinstitucionales, atendiendo también a las diferentes modalidades: escuelas rurales, urbanas, suburbanas, de sectores medios, populares, contextos de encierro, domiciliarias, hospitalarias y otras. Los Institutos Formadores deberán arbitrar los medios para que tanto los Profesores de la Formación Docente como los 'maestro/as orientadores' participen en el acompañamiento, supervisión y evaluación del proceso y de la práctica propiamente dicha (DCFD, 2008, p. 33).

Como puede leerse en este fragmento, las prácticas docentes deben realizarse en diversas escuelas -incluidas las instaladas en el ámbito rural-, especificación que se reitera al presentar la propuesta para Campo de la Práctica de $2^{\circ}, 3^{\circ}$ y $4^{\circ}$ año de la carrera. Por ejemplo, al desglosar los contenidos asignados a $2^{\circ}$ año, el DCFD plantea la identificación y caracterización pedagógica, social, cultural y organizativa de instituciones escolares en diferentes contextos (urbanos, suburbanos y rurales). El ingreso al ámbito rural se propone como uno de los contextos socioculturales en que se instalan las escuelas, sin embargo, no se incluyen referencias específicas del abordaje didáctico del aula plurigrado dentro de los contenidos mínimos del resto de las materias del plan de formación. En relación con los Trayectos Formativos Opcionales, la decisión de ofrecer espacios que permitan adentrarse en la problemática particular de la escuela rural queda a criterio de cada institución.

A pesar de su inclusión en el DCFD, el contexto rural es desatendido por la mayor parte de los ISFD instalados en zonas urbanas; de este modo, se acentúa la formación de los futuros docentes en el modelo pedagógico del aula estándar (Terigi, 2008). $\underline{\underline{21}}$ Ahora bien, dado que el DCFD plantea que las prácticas docentes deben realizarse no sólo en ámbitos urbanos sino también en ámbitos rurales, resulta legítimo preguntarse acerca de quiénes estarían en condiciones de brindar tal formación específica y en qué consistiría.

En un informe presentado por el MECyT -coordinado por Patricia Sadovsky- denominado "La enseñanza de la matemática en la formación docente para la escuela primaria” (MECyT, 2010) se analizan las percepciones de los profesores a cargo de este área en los institutos de formación docente de la Argentina. Entre otros asuntos, el documento presenta un detalle de los temas solicitados por los profesores para ser abordados en instancias de capacitación que los tengan como destinatarios. De un total de 148 respuestas, solo 3 reclaman formación sobre didáctica de la matemática en plurigrados (cuadro 20, p. 82). La escasa presencia de este pedido podría llevar a concluir que se trata de un tema poco relevante, o bien, podría ser una señal de la escasa visibilización de la particularidad del plurigrado y de la necesidad de su abordaje didáctico en el tramo de la formación inicial.

\section{Formación docente continua}

En este apartado se reseñan algunas de las propuestas implementadas en el marco de la formación docente continua que contemplan la enseñanza en aulas plurigrado de escuelas rurales desde la jurisdicción provincial y nacional.

En la provincia de Buenos Aires se implementó el curso de capacitación "Hacia una mejor calidad de la educación rural” (2000-2006) con la intención de capacitar a maestros y directivos de $1^{\mathrm{o}}$ y $2^{\mathrm{o}}$ ciclos de la EGB de escuelas rurales - continentales o de islas- de la provincia de Buenos Aires. $\underline{22}$ El carácter semipresencial de la propuesta intentó responder a las singulares condiciones del trabajo del docente rural: las tareas no presenciales resolvieron las dificultades manifestadas por los maestros para asistir a las clases 
(distancias, traslados y tránsito de los caminos) y los encuentros presenciales buscaron promover los intercambios con el capacitador-tutor y entre los docentes participantes.

El curso se estructuró alrededor de documentos de apoyo para cada una de las áreas (Lengua, Matemática, Ciencias Naturales y Ciencias Sociales). La primera edición de estos materiales se remonta al año 2002, antes de la promulgación de la Ley 26.206 y la Ley 13.688. Este proyecto se ha constituido en uno de los principales referentes de la formación continua pensada como una política universal específica para los docentes rurales. $\underline{23}$ A modo de ejemplo, a continuación, se mencionan algunos de los aspectos planteados por el módulo destinado al área de Matemática.

Los autores de este módulo advierten que las investigaciones acerca de la enseñanza de la Matemática se han centrado en las aulas urbanas donde los alumnos, diversos en muchos aspectos, pertenecen al mismo grado de la escolaridad. Ante la escasez de producción didáctica acerca de los plurigrados de escuelas rurales, este curso se presentó como una oportunidad para avanzar en el análisis y la mejora de las prácticas de enseñanza en estos contextos. Los aspectos abordados intentaron responder a la complejidad de la planificación, la selección y la secuenciación de contenidos de enseñanza, los agrupamientos de alumnos, las intervenciones docentes y la evaluación.

El documento incluye el análisis didáctico de registros de clases y de secuencias de enseñanza implementadas en aulas plurigrado. Las actividades destinadas a los alumnos incluidas en los módulos presentan variantes según niveles de complejidad (nivel 1, nivel 2 y nivel 3). Esta denominación apuntó a desviar la exclusividad de la asignación de contenidos y actividades en función de la distribución graduada pautada por el Diseño Curricular. Con la misma intención, el documento propone ampliar los criterios de edad y grado que suelen comandar la organización de los agrupamientos y los intercambios entre alumnos; en su lugar, invita a considerar los conocimientos que los niños tienen disponibles.

El documento destaca también la potencia que el aula plurigrado puede representar para la enseñanza y el aprendizaje:

El plurigrado es un lugar privilegiado para que el docente pueda ser testigo de la evolución de los alumnos respecto de sus procesos de aprendizaje, ya que permanecen a su cargo durante varios años. Por otra parte, la interacción entre alumnos que enfrentan diferentes niveles de avance en la construcción de cierto contenido estimula a quienes van detrás y contribuye al afianzamiento de los que marchan delante (DGCyE, 2005, p. 220).

A su vez, reconoce el aporte que los docentes rurales pueden realizar a la reflexión sobre la atención a la diversidad, señalamiento que enriquece el sentido de las investigaciones que pueden emprenderse en estas escuelas.

A nivel nacional, interesa mencionar el "Programa Mejoramiento de la Educación Rural” (PROMER), destinado a docentes rurales de todos los niveles. Así lo describe María Rosa Brumat (2011):

A partir de 2006, y para dar cumplimiento a lo expresado en la Ley de Educación Nacional (art. 49) y a la Ley de Financiamiento Educativo $N^{\circ} 26.075$, se comienza a implementar, desde el Ministerio de Educación de la Nación, el Programa de Mejoramiento de la Educación Rural (PROMER) dirigido a todas las escuelas rurales del país. Este Programa tiene por finalidad respaldar la política del gobierno nacional orientada a mejorar la cobertura, eficiencia, calidad y gestión del sistema educativo en el sector rural, apuntando a generar acciones de política educativa para la Educación en contextos rurales (p. 3).

En la Provincia de Buenos Aires el PROMER se ha constituido en un referente significativo para los 
docentes rurales. Su actividad principal se articula en torno a encuentros provinciales en los que participan maestros, directivos e inspectores.

Una de las acciones desarrolladas en forma articulada con el PROMER se vincula con el Postítulo de Especialización en Educación Rural para el Nivel Primario (Resol.57/CFE/08). Esta especialización fue impulsada por el MECyT, diseñada y gestionada por el Instituto Nacional de Formación Docente (INFD) y la Subsecretaría de Equidad y Calidad (Resol.24/CFE/07). El dictado de los postítulos se coordinó con los ISFD que estaban autorizados para asumir esta tarea (Resol.151/CFE/00). En colaboración con la Coordinación Nacional de Educación Rural, se elaboraron diversos materiales que fueron utilizados en estas instancias formativas, entre ellos: "La educación en contextos rurales" y "Orientaciones para seminarios de profundización en Educación Rural”. $\underline{24}$ El postítulo ofrecido por el INFD entre 2008 y 2013 ha representado una de las escasas oportunidades formativas para los docentes rurales del país. Tal como disponía la misma normativa, los postítulos fueron aprobados para una cantidad determinada de cohortes y no volverían a implementarse de no mediar una investigación evaluativa. $\underline{25}$

Por último, el MECyT inició en 2013 el “Apoyo Socioeducativo a Escuelas de Personal Único” con la intención de promover mejoras en las propuestas de enseñanza específicas del plurigrado. A partir de 2015 se amplió la cobertura de escuelas, incluyendo a instituciones educativas de hasta tres secciones de grados agrupados. Desde entonces, esta acción se denomina: “Apoyo Socioeducativo a Escuelas de Personal Único y Grados Agrupados”. $\underline{26}$ Son responsables de la implementación de estas acciones la Dirección Nacional de Políticas Socioeducativas y la Modalidad Educación Rural de la Dirección Nacional de Gestión Educativa.를

Las propuestas formativas que asuman la particularidad del contexto rural deben enfrentar la doble tarea de considerar a la escuela rural en su carácter de potencial destino profesional de los futuros docentes y de realidad actual para quienes ya se desempeñan en estos contextos. Si bien los marcos normativos y curriculares han avanzado en introducir el contexto rural y la enseñanza en plurigrado, son aún escasos los ISFD y las instancias de formación continua que los contemplan.

\section{Condiciones de trabajo del docente rural}

Tal como se mencionó anteriormente, entre las características que distinguen el trabajo docente en escuelas rurales unitarias suelen señalarse el aislamiento y la soledad. $\underline{28}$ Esta condición particular reduce significativamente la frecuencia de los intercambios con otros maestros. En vistas a contrarrestar el aislamiento, los organismos oficiales nacionales y provinciales han puesto en marcha distintas acciones, entre las que se encuentran los agrupamientos de escuelas rurales (DGCyE, 2009) y la organización de encuentros periódicos entre docentes. Otro aspecto saliente se vincula con la multiplicidad de tareas (pedagógicas, de gestión, administrativas, de limpieza, comunitarias y de salud) a cargo de los maestros que trabajan en estas escuelas. La cantidad y diversidad de funciones que desempeñan suelen disminuir el tiempo destinado a la enseñanza. El incremento de las responsabilidades de estos maestros también proviene de la escasez y distancia de Jardines de Infantes en el ámbito rural. Es habitual que la escuela primaria incorpore niños pequeños para garantizar el cumplimiento de la obligatoriedad pautada por la normativa. $\underline{29}$ Asimismo, en ocasiones, asume la tarea de alfabetización de los adultos de la zona. Los rasgos particulares de la tarea del maestro rural son abordados en escasas ocasiones por la formación docente; esto dificulta el desempeño laboral.

En relación con la ocupación del cargo de maestro rural se han identificado dos fenómenos contrapuestos. $\underline{30}$ Los maestros que inician su carrera profesional encuentran en estos contextos su primera oportunidad laboral, dado que sus colegas con mayor antigüedad optan por destinos cercanos a sus domicilios. Esta 
situación resulta compleja en tanto los maestros recién graduados llegan a estas escuelas portando una formación inicial que no les ha brindado herramientas específicas para desempeñarse en el puesto para el que los habilita. En nuestro país, esta realidad ha sido asumida por el MECyT dentro del programa de acompañamiento de docentes noveles. Parte de este trabajo de desarrollo profesional ha sido difundido a través de la publicación de relatos de experiencias de los maestros rurales y los acompañantes (profesores de ISFD) que participaron de este proyecto en la provincia de Tucumán. El documento coordinado por Beatriz Alen se denomina “Iniciarse como docente en escuelas rurales” (MECyT, 2009).

El fenómeno inverso refleja la decisión de maestros que han acumulado antigüedad docente en escuelas urbanas y buscan finalizar su carrera profesional en una escuela rural, con la intención de obtener una mejora salarial a partir de la asignación por ruralidad. $\frac{31}{E}$ El docente que allí llega con la idea de una jubilación cercana trae consigo -y traslada sin grandes cambios- el capital de su formación y su experiencia de escuela urbana de sección única.

Si bien la movilidad del plantel docente también se produce en las escuelas urbanas, el impacto es aún mayor en la vida institucional de las escuelas rurales de personal único. La dificultad para comunicar al nuevo docente los proyectos de enseñanza y las historias de aprendizaje de los alumnos pone en riesgo la pretensión de continuidad.

\section{Reflexiones finales}

En estas páginas hemos abordado la particularidad y complejidad de la educación rural señalando el desafío que representa para las políticas educativas; la producción pedagógica, didáctica y curricular; el trabajo de los maestros y la formación docente en nuestro país.

Sin bien queda aún pendiente la elaboración de políticas educativas que den respuesta a la totalidad de las problemáticas y necesidades de la educación rural identificadas en las normativas y documentos reseñados, es posible reconocer avances significativos que se han producido sobre esta temática en los últimos años. En algunas ocasiones, las políticas implementadas permitieron legitimar ciertas experiencias -muchas de las cuales fueron gestadas en las propias instituciones- que representaban una ruptura de la mirada homogeneizadora de la escuela; en otras, permitieron poner en tensión la naturalización de la escuela graduada urbana como único modelo y ofrecer un marco normativo que habilite (no solo en las escuelas rurales) la transformación de las prácticas vigentes.

Entre las problemáticas que se reiteran en los diversos materiales relevados se encuentran el aislamiento y la soledad del maestro rural. Los agrupamientos de escuelas se hallan entre las acciones promovidas desde los organismos centrales de gestión educativa para contrarrestarlos; habilitan y amplían el intercambio de experiencias y el trabajo colaborativo entre alumnos, docentes y supervisores.

Con la misma intención de reducir el aislamiento, se promueve el acercamiento entre las escuelas rurales y los institutos formadores. Una de las líneas de trabajo del INFD apunta al acompañamiento de los docentes que inician su trayectoria profesional en escuelas rurales. A su vez, entendemos que la inclusión de estas escuelas como destino de la residencia docente de los estudiantes del profesorado abre un nuevo espacio para los intercambios entre formadores, estudiantes y maestros orientadores. En relación con estos espacios, resulta necesario analizar con mayor detalle la recuperación, circulación y validación de los saberes de los maestros rurales que se vehiculizan no solo a través de las observaciones de clases sino también a partir de las orientaciones que ofrecen a profesores y estudiantes al momento de elaborar las planificaciones para la residencia.

Otra problemática recurrente en el ámbito rural se vincula al ingreso tardío y a la discontinuidad de la 
asistencia ocasionada por las distancias, las condiciones climáticas, las dificultades de acceso, la incorporación a trabajos temporarios junto a sus familias y el cuidado de hermanos menores. Como mencionamos, la Resolución 174/CFE/12 y el Régimen Académico del Nivel Primario de la Provincia de Buenos Aires se encuentran entre las políticas educativas que han avanzado en dar respuesta a esta realidad que afecta al sistema educativo en su conjunto ofreciendo un marco normativo que procura sostener las trayectorias educativas de los alumnos.

En los materiales relevados también se reconoce la complejidad del trabajo del maestro a cargo de escuelas unitarias, asociada fundamentalmente a la multiplicidad de tareas a su cargo y a los desafíos de gestionar la enseñanza en aulas plurigrado. La escasez de producción pedagógico-didáctica específica y la insuficiencia (o ausencia) de su abordaje desde las instancias formativas se señalan como factores que incrementan tal complejidad. A partir de este reconocimiento, se promueven: la producción de materiales específicos, la incorporación de su estudio en instancias de formación docente inicial y continua y la oferta de postítulos.

Entre los avances que se han realizado en este sentido podemos mencionar, a nivel nacional, la creación de la modalidad Educación Rural a partir de la Ley 26.206, el "Programa Mejoramiento de la Educación Rural” (PROMER) y la creación por parte del INFD del postítulo "Especialización Superior en Educación Rural para Nivel Primario". Las acciones de capacitación desarrolladas por el PROMER y el INFD apuntan a proporcionar herramientas didácticas y pedagógicas para el trabajo en aulas plurigrado y a generar espacios de trabajo grupal que minimicen el aislamiento propio del contexto rural. Interesa señalar que el PROMER se ha constituido en referente clave para los docentes rurales de la provincia de Buenos Aires. Sin embargo, llama la atención que, a pesar del alto porcentaje de escuelas rurales de esta provincia, no se haya incorporado la Orientación en Educación Rural dentro de las especializaciones que se ofrecen desde los ISFD y que no figure dentro de las jurisdicciones que han participado del Postítulo en Educación Primaria Rural organizado por el INFD. Si bien no estamos en condiciones de dar cuenta de las razones que fundamentan estas decisiones, lo señalamos como un tema interesante a indagar en futuros estudios y como un ejemplo del impacto que tienen las políticas educativas (asumidas o desestimadas) sobre el sistema educativo, en este caso particular, sobre la formación docente.

A nivel provincial, destacamos la incorporación del contexto rural en el marco de la Ley 13.688 y del Diseño Curricular para la Formación Docente. Como ha sido mencionado, estos dos hitos significativos recuperan y capitalizan experiencias previas de capacitación docente desplegadas en Buenos Aires como, por ejemplo, el curso "Hacia una mejor calidad de la educación rural” implementado entre 2000 y 2006. Si bien la presencia explícita del contexto rural en la propuesta curricular del Nivel Superior representa sin dudas un avance significativo, no podemos dejar de señalar algunas cuestiones aún pendientes y resaltar, una vez más, la necesidad de contemplar la trama compleja que es necesario adaptar o transformar para que las políticas educativas se implementen desplegando toda su potencialidad. Entre las cuestiones pendientes se encuentran: las dificultades para organizar y supervisar las observaciones y las prácticas docentes de los estudiantes en las escuelas rurales, la escasa disponibilidad de materiales de consulta y la falta de formación de los profesores sobre el contexto rural y la enseñanza en plurigrado. En la mayoría de los ISFD estas dificultades han sido interpretadas como impedimentos o justificativos que eximen del cumplimiento de la prescripción curricular, profundizando aún más la formación de los futuros docentes en un único modelo pedagógico: el del aula graduada urbana de sección única.

Para finalizar, interesa explicitar que el Diseño Curricular para la Educación Primaria vigente desde 2008 establece una propuesta única para el universo de escuelas de la provincia, incluidas las rurales. Los países de la región han transitado diversos recorridos que avanzan hacia (o retornan de) la elaboración de diseños curriculares adaptados o específicos para la educación rural. Lo destacamos como otro de los ejes centrales que las políticas educativas vinculadas a la educación rural deben considerar. 


\section{Notas}

1 Datos suministrados por el Departamento de Establecimientos Educativos de la Dirección Provincial de Educación Primaria de la provincia de Buenos Aires el 17/2/16.

$\underline{2}$ Datos recuperados de www.abc.gov.ar en 20/1/16. No se informa el porcentaje de escuelas unidocentes y bidocentes de la provincia de Buenos Aires.

$\underline{3}$ Escobar, M. (2016). "La enseñanza de la Matemática en aulas plurigrado. Un estudio de caso sobre un Instituto Superior de Formación Docente de la provincia de Buenos Aires”. Tesis de Maestría en Educación dirigida por la Dra. Claudia Broitman. Fahce. UNLP.

4 Para referirse a este tipo de escuelas suele usarse la denominación de escuela unidocente, unitaria o de personal único (PU).

$\underline{5}$ Este artículo de la Ley 1.420 puede ser considerado como un antecedente de la tendencia a circunscribir los contenidos de enseñanza a los contextos en que se instalan las escuelas. Si bien el propósito manifiesto se apoya en el respeto por lo local y la promoción del arraigo a la comunidad de origen y a la tierra, algunos autores denuncian que subyace a estas ideas la intención de evitar el incremento de la población urbana ocasionada por las migraciones internas provenientes de la zona rural (Prof. Vital. Coordinadora del Fondo bibliográfico Maestro Luis F. Iglesias. Universidad Nacional de Luján. Recuperado el 30/1/16 de: http://www.fbluisiglesias.unlu.edu.ar/)

6 La Ley 24.195 estableció una nueva estructura para el sistema educativo que fue asumida por la provincia de Buenos Aires. La obligatoriedad se extiende desde la sala de 5 años hasta el $9^{\circ}$ año de la EGB (art. 10). La EGB se organiza en tres ciclos: $1^{\circ}$ ciclo $\left(1^{\circ}, 2^{\circ}\right.$ y $3^{\circ}$ años $), 2^{\circ}$ ciclo $\left(4^{\circ}, 5^{\circ}\right.$ y $6^{\circ}$ años $)$ y $3^{\circ}$ ciclo $\left(7^{\circ}, 8^{\circ}\right.$ y $9^{\circ}$ años). El Nivel Polimodal es de tres años de duración. Si bien no se desarrollará en esta oportunidad, es importante señalar que esta estructura generó un desafío administrativo, organizacional y académico para el ámbito rural (Záttera, 2015).

$\underline{7}$ El Área de Educación Rural se desarrolla en el marco de la Dirección Nacional de Gestión Curricular y Formación Docente. En el mismo informe se aclara que esta área toma como punto de partida las políticas compensatorias del MECyT que atendieron a la ruralidad desde la década de los 90 hasta la creación del Área de Educación Rural en 2004 y la Modalidad Educación Rural en 2006.

8 La Ley 24.195 alude a la gradualidad en dos de sus artículos, reemplazando la denominación de "grado" por la de “año”. Esta modificación incurre en la ambigüedad de utilizar una misma denominación para referirse a la edad de los alumnos y al tramo de la escolaridad que cursan. Al amparo de esta ley, numerosas provincias dejaron de lado la expresión "grado" para referirse a los tramos en que se divide la enseñanza básica o primaria. En su reemplazo, se ha popularizado la expresión "año", aunque no de modo homogéneo en diferentes provincias y regiones del país (Ideas tomadas de la exposición de Isabelino Siede durante el Simposio Interno de Investigación realizado en la Fahce, UNLP, 2015).

9 El Informe presentado en 2004 profundiza la flexibilización habilitada por la Ley 24.195 desde 1993: "Los niveles, ciclos y regímenes especiales que integren la estructura del sistema educativo deben articularse, a fin de profundizar los objetivos, facilitar el pasaje y continuidad, y asegurar la movilidad horizontal y vertical de los alumnos/as. En casos excepcionales, el acceso a cada uno de ellos no exigirá el cumplimiento cronológico de los anteriores sino la acreditación, mediante evaluación por un jurado de reconocida competencia, de las aptitudes y conocimientos requeridos” (art.12).

10 Terigi (2006) describe los principales propósitos de estas iniciativas, entre las que se refiere a los grados de aceleración implementados en la Ciudad Autónoma de Buenos Aires. 
$\underline{11}$ La Ley 26.206 propone una nueva estructura para el sistema educativo. El Nivel Inicial está organizado en dos ciclos: el $1^{\circ}$ ciclo (45 días a sala de 2 años) y $2^{\circ}$ ciclo $\left(1^{\circ}, 2^{\circ}\right.$ y $3^{\circ}$ sección definidas por edad 34 y 5 años respectivamente). El Nivel Primario organizado en dos ciclos: $1^{\circ}$ ciclo $\left(1^{\circ}, 2^{\circ}\right.$ y $3^{\circ}$ años $)$ y $2^{\circ}$ ciclo $\left(4^{\circ}, 5^{\circ}\right.$ y $6^{\circ}$ años). El Nivel Secundario de seis años de duración se declara obligatorio.

12 Záttera resalta que "la diferencia entre el mínimum de instrucción para las escuelas urbanas y rurales de la Ley 1.420 se reemplaza por la prescripción de equivalencia” (Záttera, 2015, p.27).

13 La Ley 13.688 define diferentes niveles, ámbitos (rurales continentales y de islas, urbanos, contextos de encierro, virtuales, domiciliarios y hospitalarios) y modalidades (Educación Técnico Profesional, Educación Artística, Educación Especial, Educación Permanente de Jóvenes, Adultos, Adultos Mayores y Formación Profesional, Educación Intercultural, Educación Física, Educación Ambiental y Psicología Comunitaria y Pedagogía Social) para el sistema educativo provincial (art. 21). A diferencia de la LEN, la ley provincial no adopta la Modalidad Educación Rural sino que lo rural es considerado como uno de los ámbitos de desarrollo (cap. XVII).

14 El documento fue presentado para el debate a través de la Res.109/CFE/10 y aprobado por la Res.128/CFE/10.

$\underline{15}$ Es importante aclarar que a principios del año 2016 se introdujeron modificaciones a esta normativa. En este artículo hacemos referencia a la versión original aprobada en 2014 vigente durante la investigación. Futuros estudios podrán evaluar el impacto de estas modificaciones en la educación rural.

16 Si bien se reconoce que la atención a la diversidad, planteada desde una perspectiva intercultural, se opone al supuesto de homogeneidad y pone de relieve la multiplicidad de identidades que atraviesan las aulas (étnicas, de género, culturales, sociales, lingüísticas, etc.); usamos el término “diversidad” para aludir a la consideración simultánea de contenidos de enseñanza y de conocimientos y ritmos de aprendizaje de los alumnos correspondientes a diferentes grados de la escolaridad.

$17 \mathrm{Si}$ bien su estructura es ciclada, la escolaridad es graduada. Incluso en las escuelas rurales de aula plurigrado, donde alumnos de diversos grados comparten el espacio del aula y el docente, siguen estando matriculados en un grado determinado promoviendo o no al grado siguiente al finalizar el ciclo lectivo.

18 Según datos de 2010, Argentina cuenta con 10.325 escuelas primarias rurales estatales que representan el 46,35\% del total de establecimientos de Nivel Primario del país. Del total de escuelas rurales, 3.409 son unidocentes y representan 33\% de aquellas (DINIECE, 2010 citado en Terigi, 2013).

$\underline{19}$ Este documento integra una serie que incluye orientaciones para diferentes modalidades del sistema educativo elaboradas por el Instituto Nacional de Formación Docente (INFD) del MECyT.

$\underline{20}$ Se denomina "maestro orientador" al docente a cargo del aula en que los estudiantes realizan las prácticas docentes.

$\underline{21}$ Terigi distingue entre modelo pedagógico y modelo organizacional. Plantea que frente a distintos modelos organizacionales (sección única, plurigrado, clase de apoyo) se cuenta con un único modelo pedagógico, el del aula estándar (escuela urbana graduada de sección única). Los docentes que se desempeñan frente a distintos modelos organizacionales, extienden los saberes docentes ligados al único modelo pedagógico del que disponen.

$\underline{22}$ El curso de capacitación fue desarrollado desde la Dirección Provincial de Educación Superior y de Formación y Capacitación Docente Continua (DGCyE).

$\underline{23}$ Los contenidos desarrollados en el documento son consistentes con el Diseño Curricular para la 
Educación General Básica (1999) -vigente en aquel momento- y con una serie de publicaciones de la DGCyE (2001) sobre el área de Matemática.

24 Materiales disponibles en: http://www.me.gov.ar/infod

$\underline{25}$ La evaluación de este postítulo puede consultarse en el documento "Evaluación de la especialización docente de nivel superior en Educación Rural para el nivel primario. Informe final 2010”. Según este informe, la provincia de Buenos Aires no se encontraba entre las 20 provincias que habían participado de la oferta del postítulo en Educación Rural. Disponible en: http://cedoc.infd.edu.ar

$\underline{26}$ Información recuperada el 10/1/2016 de: http://portales.educacion.gov.ar/dnps/apoyo-a-escuelas-depersonal-unico/

$\underline{27} \mathrm{Al}$ momento de realizar nuestro estudio la Dirección Nacional de Políticas Socioeducativas y la Modalidad de Educación Rural dependían del MECyT. A partir del cambio de gestión producido en 2016 pasan a depender del MEyD.

$\underline{28}$ Plantear el aislamiento profesional como un rasgo de la escuela rural puede tender a la invisibilización de este fenómeno como problema de la escuela urbana. Es compartido por la literatura educativa que no resulta suficiente que en una misma institución se reúna un gran número de docentes para que se produzca el trabajo colaborativo. Lortie (1975, citado en Escobar, 2012) identifica el individualismo como una de las características de la cultura profesional docente, favorecido por la arquitectura y la organización del espacio y del tiempo escolar.

$\underline{29}$ Este tipo de agrupamiento, también denominada aula multinivel, no solo reúne alumnos que cursan grados diferentes sino que pertenecen a distintos niveles del sistema educativo.

$\underline{30}$ El título docente otorgado por los ISFD habilita a los maestros a desempeñarse tanto en escuelas urbanas como rurales. Como mencionamos, la postitulación en Educación Rural no resulta un requisito excluyente.

$\underline{31}$ Los docentes que se desempeñan en escuelas ubicadas en zona rural reciben un incremento salarial detallado en el Capítulo VIII (art. 31 y 36) del Estatuto del Docente de la provincia de Buenos Aires. Entre los criterios para determinar tal asignación se consideran la ubicación de la escuela, la distancia a recorrer, el estado de los caminos, la disponibilidad de transporte público (Capítulo III, art. 10).

\section{Bibliografía}

Brumat, M. R. (2011). Maestros rurales: condiciones de trabajo, formación docente y práctica cotidiana. En Revista Iberoamericana de Educación, 55(4),1-10. Recuperado de http://rieoei.org/deloslectores/3939Brumat.pdf

Dussel, I. (2006). De la primaria a la EGB: ¿qué cambios en la enseñanza elemental en los últimos años? En Terigi, F. (comp.). Diez miradas sobre la escuela primaria (pp. 85-130) Buenos Aires: Fundación OSDE/Siglo XXI.

Quaranta, M. E., y Wolman, S. (2003). Discusiones en las clases de matemáticas. Qué, para qué y cómo se discute. En Panizza, M. (comp) Enseñar Matemática en el Nivel Inicial y primer ciclo de EGB: Análisis y Propuestas (pp. 189-243) Buenos Aires: Paidós.

Terigi, F. (2004). La enseñanza como problema político. En Frigerio, G. y Diker, G. (comps.) La transmisión en las sociedades, las instituciones y los sujetos. Un concepto de la educación en acción (pp.191-202). Buenos Aires: Novedades Educativas.

Terigi, F. (2006). Las ‘otras’ primarias y el problema de la enseñanza. En Terigi, F. (comp.) Diez miradas 
sobre la escuela primaria (pp.191-230). Buenos Aires: Fundación OSDE/Siglo XXI.

Terigi, F. (2008). Organización de la enseñanza en los plurigrados de escuelas rurales. Tesis de Maestría. Buenos Aires: FLACSO.

Záttera, O. (2015). Enseñar y aprender en el campo. Reflexiones sobre el sistema educativo en el ámbito rural. Lomas de Zamora: Editorial UNLZ, Facultad de Ciencias Sociales.

\section{Legislación y documentos consultados}

Argentina. Ministerio de Educación, Ciencia y Tecnología de la Nación (2004). Informe en el Seminario "Educación de la población rural en América Latina: alimentación y educación para todos". Santiago de Chile: UNESCO-FAO. Recuperado de: http://www.me.gov.ar/curriform/publica/edrural.pdf

Argentina. Ministerio de Educación, Ciencia y Tecnología de la Nación (2009). Iniciarse como docentes en escuelas rurales. Serie Acompañar los primeros pasos en la docencia. Recuperado de: http://www.bnm.me.gov.ar/giga1/documentos/EL003095.pdf

Argentina. Ministerio de Educación, Ciencia y Tecnología de la Nación (2010). La enseñanza de la matemática en la formación docente para la escuela primaria. Serie Estudios Nacionales. Recuperado de: http://cedoc.infd.edu.ar/upload/Investigacion nacional SADOVSKY .PDF

Argentina. Dirección General de Cultura y Educación (2005). Hacia una mejor calidad de la educación rural: Matemática. Escuelas rurales. $1^{\circ}$ y $2^{\circ}$ ciclo de la EPB. Documentos de apoyo para la capacitación. Buenos Aires.

Argentina. Dirección General de Cultura y Educación (2008). Diseño Curricular para la Educación Superior. Nivel Inicial y Nivel Primario. Buenos Aires.

Argentina. Dirección General de Cultura y Educación (2009). Documento $N^{\circ}$ 1: El agrupamiento como herramienta estratégica para el desarrollo rural e islas. Buenos Aires. 